\title{
Totally laparoscopic approach for a single mesenteric localization of Castelman's disease: state of the art of laparoscopic surgery
}

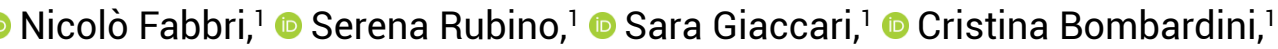 \\ - Giorgia Santandrea, ${ }^{1}$ (1) Luigi Romeo, ${ }^{1}$ ㄴ Franceca Petrarulo, ${ }^{1}$ \\ Massimo Pedriali, ${ }^{2}$ [C Gabriele Anania ${ }^{1}$ \\ 'Department of Morphology, Experimental Medicine and Surgery, Section of General and Thoracic Surgery, University of Ferrara, Ferrara, Italy \\ ${ }^{2}$ Operative Unit of Pathological Anatomy, Oncology and Specialist Medical Department, S. Anna Hospital, University of Ferrara, Italy
}

\begin{abstract}
Castleman's disease is a rare proliferative disorder of benign nature characterized by hyperplasia of the lymphoid follicles. The abdomen is rarely affected by this pathology. The difficult preoperative diagnosis has recently led laparoscopic surgery to become an option for diagnosis and treatment of the unicentric form of the disease. Herein we present a literature review reporting our experience representing, according to our knowledge, one of the first cases of totally laparoscopic resection of single abdominal localization of Castelman's disease.

Keywords: Castleman's disease; laparoscopy; surgery.
\end{abstract}

\section{Introduction}

Castleman's Disease (CD) represents a distinct clinic-pathological entity, first described by Dr. Benjamin Castleman in 1956, ${ }^{[1]}$ which still remains a rare and poorly understood disease characterized by massive growth of lymphoid tissue. A variety of terms have been used to describe this disorder, including giant lymph node hyperplasia, lymph node hamartoma, follicular lymophoreticuloma, benign giant lymphoma, angiomatous lymphoid hamartoma, and angiofollicular mediastinal lymph node hyperplasia. ${ }^{[2]}$ The most frequent location of the $\mathrm{CD}$ is the chest $(70 \%)$, although this pathology can theoretically affect any nodal station. The abdomen is rarely affected by this pathology; abdominal localizations reported in the literature are: mesentery, retroperitoneum, pancreas, pelvis and rectum. ${ }^{[3]}$

We conducted a review of the English literature articles of CD treated with laparoscopic surgery using PUBMED and Google SCHOLAR databases from the first publications until January 2018.

The words used for our research were: "Castleman's Disease" and "laparoscopy". We found 14 cases of Castleman's Disease treated with video assisted approach. It is exceptionally uncommon for Castleman's disease to present in the mesentery and only 54 cases except ours

Received: 25.06.2018 Accepted: 05.08.2018

Correspondence: Nicolò Fabbri, M.D., Via Aldo Moro 844100 Ferrara - Italy

e-mail: nicolo.fabbri@student.unife.it 
have ever been described in the literature, with 52 of them that underwent a laparotomic excision, and two cases with a laparoscopic-assisted approach. ${ }^{[4]}$

There are three pathologic variants of Castleman's Disease: hyaline vascular CD, plasma cell CD, and mixed type of $\mathrm{CD}$, which is characterized by the presence of both hyaline vascular and plasma cell CD types. Plasmablastic variant of $C D$, which is considered as a sub-variant of plasma cell type, occurs predominantly in immunosuppressed patients and human immunodeficiency virus (HIV)-positive patients. ${ }^{[5]}$ Clinic-pathologically, we can find 3 types of variants: (1) Localized CD of the HV type, presenting as an asymptomatic and slow-growing mass, (2) Localized CD of the PC type, featuring systemic manifestations of inflammation, and (3) B-cell hyperreactivity. The third group is also referred to as Multicentric Castleman's Disease (MCD) and is characterized by systemic lymphadenopathy, frequent multi-organ involvement, and more aggressive behavior. ${ }^{[6]}$ The diagnosis is difficult and the diagnostic certainty is obtained only by histological examination. Furthermore, there is no gold- standard treatment for this disease. ${ }^{[3]}$

Surgery is the optimal therapeutic approach only in the localized form, while for unresectable or disseminated disease, partial surgical resection, steroid, chemotherapy and radiotherapy have been employed with some measurable success. ${ }^{[7]}$

This report describes a case of a mesenterial localization of the hyaline vascular $\mathrm{CD}$ in the localized form.

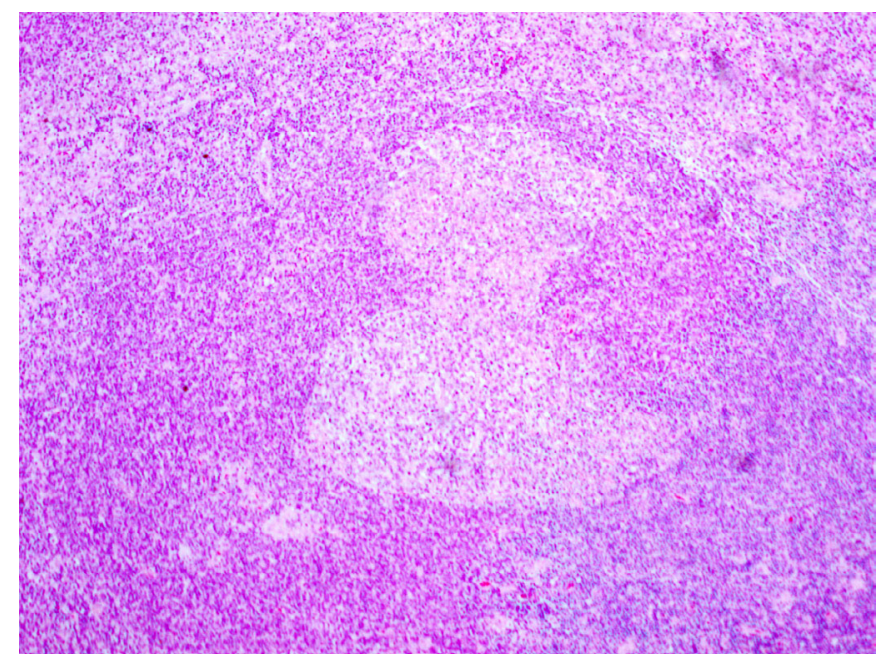

Figure 1. Castelmann's nodule: angiofollicular lymph node hyperplasia with prominent hyaline vascular lesions. Mantle zone present "onion skin" apparence. (Hematoxylin and eosin 4x).

\section{Case Report}

A.A., a 28 years old man with a history of Bechet Desease HLA B51 positive, spastic-ataxic paraparesis, treated with immunosuppressive therapy for several years, was submitted to abdominal ultrasound during investigations for a fever non responsive to drugs. The abdominal ultrasound highlighted the presence of a $4 \mathrm{~cm}$ nodule in the right iliac fossa without abdominal pain.

The Patient underwent a CT abdominal scan that shows a $4 \mathrm{~cm}$ inhomogeneus nodule near the duodenum and multiples nodules in the mesentery range. He then underwent an exploratory laparoscopy.

We used Open technique in periumbilical region for the first trocar $(10 \mathrm{~mm})$ and a $14-\mathrm{mmHg}$ pneumoperitoneum was induced. Under direct vision, three other laparoscopic accesses of $10 \mathrm{~mm}$, two in the right pararectal position, respectively above and below the umbilical, and one in the left sub-umbilical pararectal. A panoramic inspection of the abdominal cavity was carried out revealing the presence of a round mass arising in the colon trasversus mesenterium, which was first identified and then removed by means of an extraction bag. The postoperative clinical course was characterized by evacuation of faecis and gas for the two days after surgery. Three days after surgery we have noticed abdominal widespread pain and distention, with no evidence of peritonitis. We decided to replace the nasogastric tube and to perform an abdominal x-ray.

The abdominal x-ray showed a significant gaseous distention of the right colon till sigma, with air-fluid levels in

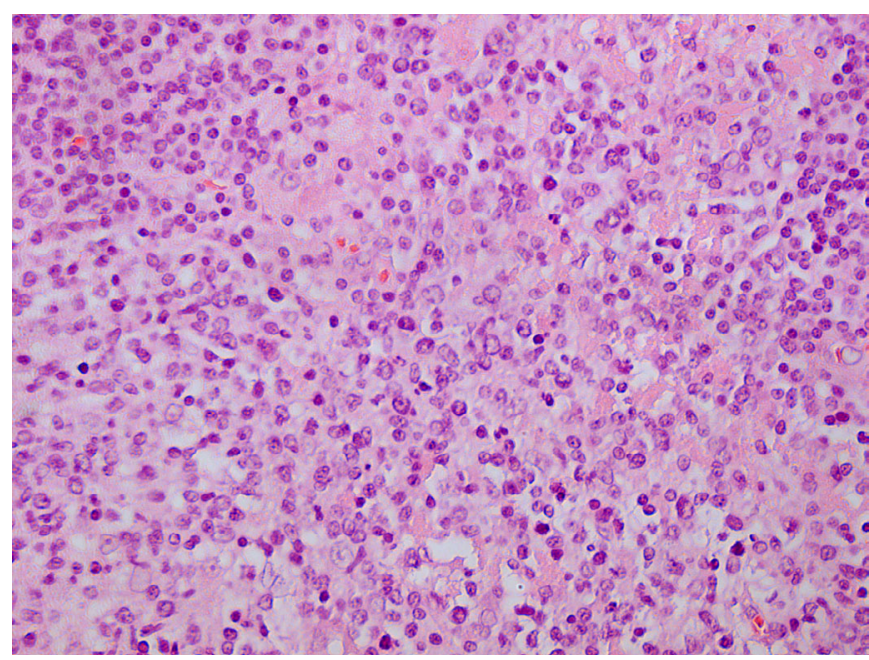

Figure 2. Castelmann's nodule: no atypical immunoblasts or plasmablasts are found. Prominent interfollicular vascular proliferation is observed. (Hematoxylin and eosin 20x). 
the small intestine. Six days after surgery, we removed the nasogastric tube and the alvus was regular. The Patient started a liquid diet, returning to a normal diet eight days after surgery. In the $9^{\text {th }}$ postoperative day the patient was discharged.

The histological diagnosis was Castelman's lymphadenopathy, hyaline vascular variant (Figs. 1, 2).

During Reumatological follow-up (last contact on September 2017), the regression of the lymphadenopathy was recorded, a low grade bi-weekly fever persisted (3-4 days lasting), until the introduction of Azatioprin $200 \mathrm{mg} /$ die. The patient was found in good conditions.

\section{Discussion}

Castleman's disease is a very rare condition that may resemble more common disease. The prevalence of CD has not been estimated, but it has been calculated that the number of cases in the United States ranges from 30,000 to 100,000 . Its incidence rate has not been reported in literature, although CD appears to be more common in the Asian population. ${ }^{[4]}$ In a series of 16 cases in 1999 from the Memorial Sloan-Kettering Cancer Center (MSKCC), patients with unicentric CD were frequently accidentally discovered with symptoms mostly arising from mass compression. ${ }^{[8]}$

In 2005, Brusciano et al. ${ }^{[9]}$ reported 330 cases of localized $\mathrm{CD}$ and about two thirds of the observed cases were localized in the mediastinum or hilus of the lung. The diagnosis is usually achieved only after surgical resection of the mass.

Currently, we have found more than 400 referred cases referred, with the localized form as the most frequent. The multicentric is the rarest variant, often related to immunosuppression and HIV infection.

CD can occur at all ages, where the majority of cases is asymptomatic (51\%), especially in the localized form, which is often accidentally diagnosed.

As our case confirms, Unicentric disease is more common in the $3^{\text {rd }}$ and $4^{\text {th }}$ decade, whereas the multicentric form is more common in the $5^{\text {th }}$ and $6^{\text {th }}$ decade with no sex predilection. ${ }^{[1]}$ Occasionally, when the lesion is large enough, compressive or constitutional symptoms may be present. It tends to occur in the third and fourth decade of life with a slight female predominance, with a median age of 35 years. ${ }^{[4]}$
Symptoms are related to the size of the affected lymph nodes (6 $\mathrm{cm}$ in average, ranging from 1 to $12 \mathrm{~cm}$ ). In this case, chest or abdominal pain may occur due to the mass compression. In $30 \%$ of cases CD results in general systemic symptoms such as asthenia, fever, and weight loss. ${ }^{[6]}$

The multicentric variant is almost always symptomatic. Symptoms are fever, generalized lymphadenopathy, hepatomegaly and/or splenomegaly, POEMS syndrome (Polyneuropathy, Organomegaly, Endocrinopathy, Monoclonal gammopathy and Skin changes), Kaposi's sarcoma. ${ }^{[6]}$

The etiology of CD is unknown, but several studies highlight the role of HHV8 in the genesis of this disorder. HIV seropositive individuals appear to be at an increased risk for multicentric Castleman's Disease at a younger age due to the increased incidence of HHV- 8 infection. ${ }^{[1]}$

HHV8 is present in $60-100 \%$ of patients with CD and HIV, but it is also present in 20-40\% in HIV negative patients. [10] The Epstein-Barr Virus, Toxoplasma and Mycobacterium tuberculosis have been considered to be involved in the genesis of some cases of variant PC CD. This disease could be a response to a chronic inflammatory process, an hamartomatous process, a state of immunodeficiency or an immune disorder. ${ }^{[3]}$

The diagnosis is based on the histological analysis of the lymph node using immunohistochemical methods.

The treatment of $\mathrm{CD}$ depends upon the type of the disease. Unicentric form is generally curable using surgical resection (with or without radiotherapy). ${ }^{[1]}$ Radiation therapy has been used with mixed success in patients who are poor surgical candidates or Castleman's Disease, those with unresectable lesions. ${ }^{[2]}$ At present, there is no consensus on the optimal management strategy for MCD. Successful treatment of MCD has been achieved using chemotherapy, with or without prednisone, given at the time of initial diagnosis. ${ }^{[1]}$

Surgery, radiotherapy, steroids, immunotherapy (interferon- $\alpha$ or anti-IL- 6 antibodies), in combination with chemotherapy have all been used to manage the disease. [11] Complete surgical excision remains the most used treatment strategy for unicentric $\mathrm{CD}$, which confers a cure rate approaching $100 \%{ }^{[12]}$ Radiologic evaluation mainly involves computed tomography (CT), magnetic resonance (MR) imaging and positron emission tomography (PET/ CT). The typical feature on CT scan is a well circumscribed 
mass with soft tissue attenuation and rarely calcification. On MR imaging, these highly vascular tumors appear solid and have intermediate to high signal compared to muscle on T1 weighted images. Hyper intense signal is seen on T2 weighted images. PET scan is a useful modality for staging and monitoring response to chemotherapy. ${ }^{[13]}$

The treatment of $\mathrm{CD}$ depends upon the variety of disease. Unicentric form is generally curable using surgical resection (with or without radiotherapy).

CD must be included in the differential diagnosis of inhomogeneus nodules in the mesentery range, especially in rheumatological patients treated with immunosuppressive therapy, even though the definitive diagnosis can only be achieved by histological and immunohistochemical examination. Therefore, in the appropriate clinical setting, the diagnosis of $\mathrm{CD}$ must be considered, after investigating and excluding more common causes of lymphadenopathy or associated neoplastic processes. In cases of masses of an uncertain nature, laparoscopy must be considered the last diagnostic tool and the first treatment.

In the case of single location, the treatment of choice is surgical removal. In our experience, the laparoscopic approach is the most appropriate choice, if not contraindicated, because it allows to explore the entire abdominal cavity, early mobilization with a reduction of postoperative pain, and a shorter duration of hospitalization.

\section{Disclosures}

Informed Consent: Written informed consent was obtained from the patient for the publication of the case report and the accompanying images.

Peer-review: Externally peer-reviewed.

Conflict of Interest: None declared.

\section{References}

1. Shah D, Darji P, Lodha S, Bolla S. Unicentric Castleman's Disease of Abdomen. J Radiol Case Rep 2013;7:26-33.
2. Wang $\mathrm{H}$, Wieczorek RL, Zenilman ME, Desoto-Lapaix F, Ghosh $\mathrm{BC}$, Bowne WB. Castleman's disease in the head of the pancreas: report of a rare clinical entity and current perspective on diagnosis, treatment, and outcome. World J Surg Oncol 2007;5:133. [CrossRef]

3. Greco LG, Tedeschi M, Stasolla S, Gentile A, Gentile A, Piscitelli D. Abdominal nodal localization of Castleman's disease: report of a case. Int J Surg 2010;8:620-2. [CrossRef]

4. Bracale U, Pacelli F, Milone M, Bracale UM, Sodo M, Merola $G$, et al. Laparoscopic treatment of abdominal unicentric castleman's disease: a case report and literature review. BMC Surg 2017; 17:38. [CrossRef]

5. Guo H, Shen Y, Wang WL, Zhang M, Li H, Wu YS, et al. Castleman disease mimicked pancreatic carcinoma: report of two cases. World J Surg Oncol 2012;10:154. [CrossRef]

6. Iwaki N, Sato Y, Takata K, Kondo E, Ohno K, Takeuchi M, et al. Atypical hyaline vascular-type castleman's disease with thrombocytopenia, anasarca, fever, and systemic lymphadenopathy. J Clin Exp Hematop 2013;53:87-93. [CrossRef]

7. Farruggia $P$, Trizzino A, Scibetta $N$, Cecchetto $G$, Guerrieri $\mathrm{P}, \mathrm{D}$ 'Amore ES, et al. Castleman's disease in childhood: report of three cases and review of the literature. Ital J Pediatr 2011;37:50. [CrossRef]

8. Bowne WB, Lewis JJ, Filippa DA, Niesvizky R, Brooks AD, Burt $\mathrm{ME}$, et al. The management of unicentric and multicentric Castleman's disease: a report of 16 cases and a review of the literature. Cancer 1999;85:706-17. [CrossRef]

9. Brusciano L, Rossetti G, Maffettone V, Napolitano V, Izzo D, Pizza F, et al. Laparoscopic Treatment of an Uncommon Abdominal Localization of Castleman Disease. Surg Laparosc Endosc Percutan Tech 2005;15:241-3. [CrossRef]

10. Cecka F, Ferko A, Jon B, Subrt Z, Kasparova P, Repak R. Pancreatic Castleman disease treated with laparoscopic distal pancreatectomy. Hepatobiliary Pancreat Dis Int 2013:12:332-4.

11. El-Osta HE, Kurzrock R. Castleman's disease: from basic mechanisms to molecular therapeutics. Oncologist 2011;16:497-511. [CrossRef]

12. Chen $\mathrm{CH}$, Liu HC, Tung KY, Lee JJ, Liu CL, Liu TP Surgical outcome of superficial and deep Castleman disease. ANZ J Surg 2007;77:339-43. [CrossRef]

13. Shetty S, Brenes RA, Panait L, Sanchez JA. Video assisted thoracoscopic resection of a posterior mediastinal Castleman's tumor. J Cardiothorac Surg 2011;6:113. [CrossRef] 